\title{
ON SOME CLASSES OF REGULAR ORDER SEMIGROUPS
}

\author{
Zhenlin Gao and Guijie Zhang
}

\begin{abstract}
Here, some classes of regular order semigroups are discussed. We shall consider that the problems of the existences of (multiplicative) inverse ${ }^{\delta}$ po-transversals for such classes of po-semigroups and obtain the following main results: (1) Giving the equivalent conditions of the existence of inverse ${ }^{\delta}$ po-transversals for regular order semigroups (2) showing the order orthodox semigroups with biggest inverses have necessarily a weakly multiplicative inverse ${ }^{\delta}$ po-transversal. (3) If the Green's relation $\mathcal{R}$ and $\mathcal{L}$ are strongly regular (see. sec.1), then any principally ordered regular semigroup (resp. ordered regular semigroup with biggest inverses) has necessarily a multiplicative inverse ${ }^{\delta}$ po-transversal. (4) Giving the structure theorem of principally ordered semigroups (resp. ordered regular semigroups with biggest inverses) on which $\mathcal{R}$ and $\mathcal{L}$ are strongly regular.
\end{abstract}

In T. S. Blyth and G. A. Pinto ([1]-[3]), the following concepts have been introduced and discussed.

A negative (resp. positive) ordered regular semigroup $S$ means that $S$ is an order semigroup (i.e. po-semigroup) in which for any $x \in S$ there is $s \in S$ such that $x s x \leq x$ (resp. $x \leq x s x$ ). A (negative) order regular semigroup $S$ is said to be principally ordered, for short POR-semigroup $S$, if for any $x \in S$ there exists

$$
x^{*}=\max \{y \in S \mid x y x \leq x\} .
$$

We refer the reader to $[1,2]$, if $\mathrm{S}$ is a POR-semigroup, then every $x \in S$ has a biggest inverse, namely the element $x^{0}=x^{*} x x^{*} \in V(x)$ (which is the inverses set of $x$ ). Thus $S$ becomes an ordered regular semigroup with biggest inverses (for short ORB-semigroup $S$ ) (see [3]). In this case we always denote the set of all biggest inverse of $S$ by $S^{0}$, i.e., $S^{0}=\left\{x^{0} \mid \forall x \in S\right\}$.

Conversely, an ORB-semigroup $S$ is necessarily not a POR-semigroup. For example, we can prove that a naturally ordered ORB-semigroup $(S, \leq)$ on which $\mathcal{R}$ and $\mathcal{L}$ are regular (see [7]) can not become a POR-semigroup. It need only to notice mapping $o: S \longrightarrow S^{o}$ denoted by $x \longrightarrow x^{o}$ is always antitone on a naturally ordered POR-semigroup $(S, \leq)$ (see [2, Theorem 3.3] and [1]).

Received September 25, 2007; Revised December 18, 2007.

2000 Mathematics Subject Classification. 06F05.

Key words and phrases. regular order semigroup, inverse ${ }^{\delta}$ po-transversals, POR-semigroups, ORB-semigroups. 
This is in contradiction with the mapping $o$ being isotone on $E(S)$ (which is the set of all idempotents of $S$ ).

Let $S$ be a po-semigroup. If $S$ is also regular (resp. orthodox, inverse and so on) then we call that $S$ is a regular (resp. orthodox,inverse and so on) posemigroup. Clearly, the POR- and ORB-semigroups are regular po-semigroups.

Let $T$ be a po-subsemigroup of the regular po-semigroup $S$. If there is a surjective mapping $\delta$ from $S$ to $T$ denoted by $x \longrightarrow x^{\delta}$ such that

(R1) $(\forall x \in S) x^{\delta} \in V_{T}(x)$ (where $\left.V_{T}(x)=V(x) \cap T\right)$

(O1) $(\forall x \in S)\left|V_{T}(x)\right|=1$ then $T$ is called an inverse ${ }^{\delta}$ po-transversal of $S$. If $T$ satisfies (R1), (O1) and

(O2) $(\forall x, y \in S) x^{\delta} x y y^{\delta} \in E(T)\left(\operatorname{resp} .\left(x^{\delta} x y y^{\delta}\right)^{\delta} \in E(T)\right)$

then $S$ is said to be multiplicative (resp.weakly multiplicative). If $T$ satisfies (R1), (O1) and

(O3) the maximum idempotent-separating congruence on $S$ is the identity congruence on $T$,

then $T$ is called a fundamental inverse ${ }^{\delta}$ po-transversal of $S$.

Clearly, any inverse ${ }^{\delta}$ po-transversal is an inverse transversal.

Let $S$ be a ORB-semigroup. Green's relation $\mathcal{R}$ and $\mathcal{L}$ are called strongly regular if (S1) and (S2) hold :

(S1) $\mathcal{R}$ and $\mathcal{L}$ are regular on $S$ (i.e. $x \leq y \Longrightarrow x x^{o} \leq y y^{o}, x^{o} x \leq y^{o} y$ )

(S2) $(\forall e, f \in E(S)) e \leq_{n} f \Longrightarrow e e^{o} \leq f f^{o}$ and $e^{o} e \leq f^{o} f$

where the order " $\leq_{n}$ " is the natural order on $E(S)$.

Example 1. Using the method in [1, Example 1], let $k>1$ be a fixed integer and for every positive integer $n$ let $n_{k}$ denote the biggest multiple of $k$ that is less than or equal to $n$. Then integer set $Z$ becomes a regular semigroup under the operation $+_{k}$ defined by $m+{ }_{k} n=m+n_{k}$; Let $(L, \wedge)$ be a semilattice and $L^{[2]}=\{(x, y) \in L \times L \mid y \leq x\}$. With the Cartesian order, let $M=L^{[2]} \times Z$. A typical element $((x, y), p)$ of $M$ will be denoted by $[x, y, p]$. Define a binary operation on $M$ by

$$
[x, y, p][a, b, q]=\left[x \wedge a, y \wedge b, p+q_{k}\right] .
$$

Then $M$ becomes a POR-semigroup and we have

$$
\begin{gathered}
{[x, y, p]^{*}=\left[x, x,-p_{k}+k-1\right]} \\
{[x, y, p]^{0}=\left[x, y,\left(-p_{k}+k-1\right)_{k}+k-1\right] ;} \\
E(M)=\left\{[x, y, p] \in M \mid \forall(x, y) \in L^{[2]}, p \in Z \text { such that } p_{k}=0\right\} .
\end{gathered}
$$

Computing we know that $E(M)$ is a left zero subband of $M$ (i.e. $E(M)$ is a subsemigroup of all idempotents of $\mathrm{S}$ ). So $M$ is orthodox. On $M$, Green's relation $\mathcal{R}$ and $\mathcal{L}$ are strongly regular. In fact, since the mapping $k: p \longrightarrow p_{k}$ 
is surjective on $Z$ and has the property : if $p \leq q$ then $p+p_{k} \leq q+q_{k}$. So we have that if $[x, y, p] \leq[a, b, q]$ then

$$
\begin{aligned}
{[x, y, p][x, y, p]^{0} } & =\left[x, y, p+\left(\left(-p_{k}+k-1\right)_{k}+k-1\right)_{k}\right] \\
& \leq\left[a, b, q+\left(\left(-q_{k}+k-1\right)_{k}+k-1\right)_{k}\right] \\
& =[a, b, q][a, b, q]^{0} .
\end{aligned}
$$

Similarly $[x, y, p]^{0}[x, y, p] \leq[a, b, q]^{0}[a, b, q]$, that is, $\mathcal{R}$ and $\mathcal{L}$ are regular. Let $[x, y, p],[a, b, q] \in E(M)$, and $[x, y, p] \leq_{n}[a, b, q]$, notice $p_{k}=q_{k}=0$ and

$$
[x, y, p]=[x, y, p][a, b, q]=[a, b, q][x, y, p]=[x, y, q]
$$

so $p=q$. Thus we have

$$
[x, y, p][x, y, p]^{0}=[x, y, p][x, y, k-1]=[x, y, p] \leq[a, b, p]=[a, b, p][a, b, p]^{0} .
$$

Similarly $[x, y, p]^{0}[x, y, p] \leq[a, b, p]^{0}[a, b, p]$, that is, (S2) holds, which shows that $\mathcal{R}$ and $\mathcal{L}$ are strongly regular.

The inverse transversal of regular semigroups was first introduced by Blyth and McFadden in [4]. In structure description of regular semigroup $S$, the inverse transversal $S^{\delta}$ play very important roles. Thus we think that the existence of an inverse ${ }^{\delta}$ transversal for a regular semigroup is of course very important problem. In this note, we study first the existence condition of inverse ${ }^{\delta}$ po-transversals. In Section 1 , we obtain necessary and sufficient conditions for a po-subsemigroup $T$ of the regular po-semigroup $S$ to be an inverse $\delta$ po-transversal. Then in Section 2, we show that any order orthodox semigroup with biggest inverses $S$ has necessarily a weakly multiplicative inverse ${ }^{\delta}$ po-transversal $S^{\delta}$ with the mapping $\delta$ is an anti-homomorphism. For an ORBsemigroup $S$ on which $\mathcal{R}$ and $\mathcal{L}$ are strongly regular then we show that $S$ has necessarily a multiplicative inverse ${ }^{\delta}$ po-transversal. Hence, in Section 3 , we give immediately the structure theorem of the POR-(resp. ORB-)semigroup $S$ on which $\mathcal{R}$ and $\mathcal{L}$ are strongly regular.

\section{The existence conditions of inverse ${ }^{\delta}$ po-transversals}

Since regular po-semigroups (resp. POR- and ORB-) are also the class of regular semigroups, so we list here some basic facts on a regular semigroup with inverse transversal used in this note, the reader can consult [4]-[7] for more details.

Lemma 1.1. Let $S$ be a regular semigroup with inverse transversal $S^{0}$. Then we have

(1) $(\forall x, y \in S)\left(x y^{o}\right)^{o}=y^{o o} x^{o}$ and $\left(y^{o} x\right)^{o}=x^{o} y^{o o}$;

(2) $(\forall x, y \in S)(x y)^{o}=\left(x^{o} x y\right)^{o} x^{o}=y^{o}\left(x y y^{o}\right)^{o}=y^{o}\left(x^{o} x y y^{o}\right)^{o} x^{o}$;

(3) $(\forall x, y \in S) x \mathcal{L} y$ if and only if $x^{o} x=y^{o} y ; x \mathcal{R} y$ if and only if $x x^{o}=y y^{o}$;

(4) $I=\left\{x x^{o} \mid x \in S\right\}$ and $\wedge=\left\{x^{o} x \mid x \in S\right\}$ are subbands of $S$;

(5) $(\forall x \in S) x^{o}=x^{o o o}$; 
(6) $S^{o}$ is multiplicative if and only if $S^{o}$ is a quasi-ideal of $S$ (i.e, $S^{o} S S^{o} \subseteq$ $\left.S^{o}\right)$ and $e^{o} \in E\left(S^{o}\right)$ for every $e \in E(S)$;

(7) If $S$ is orthodox then $e^{0} \in E(S)$ for any $e \in E(S)$ and $S^{0}$ is weakly multiplicative.

We shall give the existence conditions of inverse ${ }^{\delta}$ po-transversals for the class of regular po-semigroups.

Theorem 1.2. Let $S^{\delta}$ be a po-subsemigroup of the regular po-semigroup $S$ with the mapping $\delta$ is an anti-homomorphism from $S$ to $S^{\delta}$ denoted by $x \longmapsto x^{\delta}$ such that $x^{\delta} \in V_{S^{\delta}}(x)$. The following conditions are equivalent:

(1) $S^{\delta}$ is an inverse ${ }^{\delta}$ po-transversal of $S$;

(2) The equivalent relation

$$
\nu=\left\{(x, y) \in S \times S \mid V_{S^{\delta}}(x)=V_{S^{\delta}}(y)\right\}
$$

is the smallest inverse semigroup congruence on $S$ and the mapping $\delta \delta$ is a morphism on $S^{\delta}$.

Proof. (1) $\Longrightarrow(2)$ Let $S^{\delta}$ be an inverse ${ }^{\delta}$ po-transversal of $S$ then $S^{\delta}$ is an inverse transversal of $S$ as a regular semigroup. By Lemma 1.1 (5) $x^{\delta}=x^{\delta \delta \delta}$ for any $x \in S$. Notice that $\left|V_{S^{\delta}}(x)\right|=1$ and $x^{\delta} \in V_{S^{\delta}}(x)$ for any $x \in S$. So we have that $(x, y) \in \nu$ if and only if $V_{S^{\delta}}(x)=V_{S^{\delta}}(y)$ if and only if $V_{S^{\delta}}\left(x^{\delta \delta}\right)=V_{S^{\delta}}\left(y^{\delta \delta}\right)$ if and only if $x^{\delta}=y^{\delta}$ if and only if $x^{\delta \delta}=y^{\delta \delta}$. We denote the $\nu$-class of containing $x$ by $(x)_{\nu}$.

If $(x, y) \in \nu$ and $z \in S$ then $x^{\delta}=y^{\delta}$ and $z^{\delta} \in V_{S^{\delta}}(z)$. It follows from $\delta$ being an anti-homomorphism from $S$ to $S^{\delta}$ then $(z x)^{\delta}=x^{\delta} z^{\delta}=y^{\delta} z^{\delta}=(z y)^{\delta}$, that is, $(z x, z y) \in \nu$. Similarly $(x z, y z) \in \nu$ and thus $\nu$ is a congruence on $S$.

To show that $S / \nu$ is inverse, notice first that it is certainly regular, since any homomorphic image of a regular semigroup is regular. Now, by Lallement's Lemma in [6, II.4.6] any idempotent of $S / \nu$ is of the form e $\nu$ denoted by $e \nu=$ $(e)_{\nu}$ for $e \in E(S)$. For any $e, f \in E(S), e^{\delta} \in V_{S^{\delta}}(e), f^{\delta} \in V_{S^{\delta}}(f)$, by $S^{\delta}$ being inverse and $\delta$ being antihomomorphic we have $(e f)^{\delta}=f^{\delta} e^{\delta}=e^{\delta} f^{\delta}=(f e)^{\delta}$, that is, $(e f)_{\nu}=(f e)_{\nu}$ and $(e)_{\nu}(f)_{\nu}=(e f)_{\nu}=(f e)_{\nu}=(f)_{\nu}(e)_{\nu}$. Thus that $S / \nu$ is inverse.

Nextly, suppose that $\xi$ is an inverse semigroup congruence on $S$. Let $(x, y) \in$ $\nu$ for $x^{\delta} \in V_{S^{\delta}}(x)=V_{S^{\delta}}(y)$ and $\left(x^{\delta}\right)_{\xi} \in S / \xi$. Then it is clear that $\left(x^{\delta}\right)_{\xi}^{-1}=(x)_{\xi}$ and $\left(x^{\delta}\right)_{\xi}^{-1}=(y)_{\xi}$ by $x^{\delta}=y^{\delta}$. Thus we obtain that $(x)_{\xi}=(y)_{\xi}$, that is, $(x, y) \in \xi$. Therefore $\nu \subseteq \xi$ and so $\nu$ is as stated in (2), the smallest inverse semigroup congruence on $S$.

Finally, let $x^{\delta}, y^{\delta} \in S^{\delta}$ since $S^{\delta}$ is an inverse ${ }^{\delta}$ po-transversal of $S$ so that $x^{\delta}=y^{\delta}$ if and only if $\left(x^{\delta}\right)^{\delta \delta}=\left(y^{\delta}\right)^{\delta \delta}$ by Lemma $1.1(5)$, that is, the mapping $\left.\delta \delta\right|_{S^{\delta}}$ is a morphism.

$(2) \Longrightarrow(1)$ It need only to show that $\left|V_{S^{\delta}}(x)\right|=1$ for any $x \in S$, because this means $S^{\delta}$ is an inverse ${ }^{\delta}$ po-transversal of $S$ by Definition in Sec 1 . Let the 
equivalence relation

$$
\tau=\left\{(x, y) \in S \times S \mid x^{\delta}=y^{\delta}\right\} .
$$

Then $\tau$ is a congruence on $S$ by $\delta$ being anti-homomorphic and so $\xi=\tau \cap \nu$ is also. Clearly $(x)_{\xi} \subseteq(x)_{\nu}$ and $(x)_{\nu}=\bigcup_{y \in(x)_{\nu}}(y)_{\xi}$ for any $(x)_{\nu} \in S / \nu$. Since $S / \nu$ is inverse so there is $(y)_{\xi}^{-1} \in S / \xi$ such that $(y)_{\xi}^{-1}$ is the unique inverse of $(x)_{\xi}$ for any $(x)_{\xi} \in S / \xi$ and $y \in(x)_{\nu}$. Thus we show that $S / \xi$ is also inverse. By the smallest property of $\nu$ we have $\xi=\nu$, that is, $\nu=\tau \cap \nu$.

Now, suppose that $y^{\delta} \in V_{S^{\delta}}(x)$ then $\left(x^{\delta}\right)_{\nu}$ and $\left(y^{\delta}\right)_{\nu}$ are both inverses in $S / \nu$ of $(x)_{\nu}$. By uniqueness of inverse in $S / \nu$ we conclude that $\left(x^{\delta}\right)_{\nu}=\left(y^{\delta}\right)_{\nu}$ and so $\left(x^{\delta}\right)^{\delta}=\left(y^{\delta}\right)^{\delta}$ by $\nu=\tau \cap \nu$. Thus we imply that $x^{\delta}=\left(x^{\delta}\right)^{\delta \delta}=\left(x^{\delta \delta}\right)^{\delta}=$ $\left(y^{\delta \delta}\right)^{\delta}=\left(y^{\delta}\right)^{\delta \delta}=y^{\delta}$ by $\left.\delta \delta\right|_{S^{\delta}}$ being a morphism, which shows that $\left|V_{S^{\delta}}(x)\right|=1$ for any $x \in S$.

Corollary 1.3. Let $S^{\delta}$ be a po-subsemigroup of the ORB-(resp.POR-) semigroup $S$ with the mapping $\delta$ is an anti-homomorphism from $S$ to $S^{\delta}$ such that $x^{\delta} \in V_{S^{\delta}}(x)$ for any $x \in S$. The following conditions are equivalent;

(1) $S^{\delta}$ is an inverse ${ }^{\delta}$ po-transversal of $S$;

(2) The equivalence relation

$$
\nu=\left\{(x, y) \in S \times S \mid V_{S^{\delta}}(x)=V_{S^{\delta}}(y)\right\}
$$

is the smallest inverse semigroup congruence on $S$ and the mapping $\delta \delta$ is a morphism on $S^{\delta}$.

Theorem 1.4. Let $S^{\delta}$ be a po-subsemigroup of the regular po-semigroup $S$ with mapping $\delta$ is surjective from $S$ to $S^{\delta}$ such that $x^{\delta} \in V_{S^{\delta}}(x)$ for any $x \in S$. Then the following statements are equivalent:

(1) $S^{\delta}$ is a fundamental inverse ${ }^{\delta}$ po-transversal of $S$;

(2) The equivalent relation

$$
\mu=\left\{(x, y) \in \mathcal{H} \mid x^{\delta} e x^{\delta \delta}=y^{\delta} e y^{\delta \delta} \text { for any } e \in E\left(S^{\delta}\right)\right\}
$$

has the following properties :

$(\mu 1) \mu_{\delta}=\mu \cap\left(S^{\delta} \times S^{\delta}\right)$ is the maximum idempotent separating congruence on $S^{\delta}$ and $\mu_{\delta}$ is the identity congruence on $S^{\delta}$;

$(\mu 2) \mu$ is the smallest inverse semigroup congruence on $S$.

Proof. $(1) \Longrightarrow(2)$ Let $S^{\delta}$ be as stated in (1) and $(x, y) \in \mu$, by $(x, y) \in \mathcal{H}$ then $x x^{\delta}=y y^{\delta}$ and $x^{\delta} x=y^{\delta} y$ by Lemma 1.1 (3). Now, for any $z \in S, e \in E\left(S^{\delta}\right)$, we compute that

$$
\begin{aligned}
(z x)^{\delta} e(z x)^{\delta \delta} & =x^{\delta}\left(z x x^{\delta}\right)^{\delta} e\left(x^{\delta}\left(z x x^{\delta}\right)^{\delta}\right)^{\delta} & & (\text { by Lemma 1.1 (2)) } \\
& =x^{\delta}\left(z x x^{\delta}\right)^{\delta} e\left(z x x^{\delta}\right)^{\delta \delta} x^{\delta \delta} & & \text { (by Lemma 1.1 (1)) } \\
& =x^{\delta}\left(z y y^{\delta}\right)^{\delta} e\left(z y y^{\delta}\right)^{\delta \delta} x^{\delta \delta} & & \left(\text { by } x x^{\delta}=y y^{\delta}\right) \\
& =y^{\delta}\left(z y y^{\delta}\right)^{\delta} e\left(z y y^{\delta}\right)^{\delta \delta} y^{\delta \delta} & & \left(\text { by }\left(z y y^{\delta}\right)^{\delta} e\left(z y y^{\delta}\right)^{\delta \delta} \in E\left(S^{\delta}\right)\right) \\
& =(z y)^{\delta} e(z y)^{\delta \delta} & &
\end{aligned}
$$


that is, $(z x, z y) \in \mu$. Similarly $(x z, y z) \in \mu$. Thus we obtain that $\mu$ is a congruence on $S$ and $S / \mu$ is a regular (po-)semigroup as the proof of Theorem 1.2. By [6, V.Theorem 3.2], $\mu_{\delta}=\mu \cap\left(S^{\delta} \times S^{\delta}\right)$ is the maximum idempotentseparating congruence on $S^{\delta}$. If $S^{\delta}$ is fundamental then $\mu_{\delta}$ is the identity on $S^{\delta}$. This is the statement $(\mu 1)$.

To show the statement $(\mu 2)$ we notice that for any $(x)_{\mu} \in S / \mu,(x)_{\mu}$ contains the idempotent $x^{\delta \delta}\left(x^{\delta}\right)^{2} x^{\delta \delta} \in E\left(S^{\delta}\right)$. In fact, by $x \mathcal{L} x^{\delta} x$ and $x \mathcal{R} x x^{\delta}$ we have $x x^{\delta}=x^{\delta \delta} x^{\delta}=x^{\delta \delta}\left(x^{\delta \delta}\right)^{\delta}$ and $x^{\delta} x=\left(x^{\delta \delta}\right)^{\delta} x^{\delta \delta}$ so $x \mathcal{H} x^{\delta \delta}$. Clearly, $x^{\delta} e x^{\delta \delta}=\left(x^{\delta \delta}\right)^{\delta} e\left(x^{\delta \delta}\right)^{\delta \delta}$ by Lemma $1.1(5)$, so that $x \mu x^{\delta \delta}$ and $x \mu x^{\delta \delta} \mu$ $\left(x^{\delta \delta}\right)^{2}=\left(x^{\delta \delta}\right)^{2}\left(x^{\delta}\right)^{2}\left(x^{\delta \delta}\right)^{2} \mu x^{\delta \delta}\left(x^{\delta}\right)^{2} x^{\delta \delta}$, that is, the idempotent $x^{\delta \delta}\left(x^{\delta}\right)^{2} x^{\delta \delta} \in$ $(x)_{\mu}$. If $(x)_{\mu}$ and $(y)_{\mu}$ are idempotents of $S / \mu$ then $(x)_{\mu}=(e)_{\mu}$ and $(y)_{\mu}=(f)_{\mu}$ for some $e, f \in E\left(S^{\delta}\right)$. Thus we have

$$
(x)_{\mu}(y)_{\mu}=(e)_{\mu}(f)_{\mu}=(e f)_{\mu}=(f e)_{\mu}=(f)_{\mu}(e)_{\mu}=(y)_{\mu}(x)_{\mu}
$$

which shows that $S / \mu$ is inverse and clearly $(x)_{\mu}^{-1}=\left(x^{\delta}\right)_{\mu}$ for any $x \in S$. Now, suppose that $\xi$ is an inverse semigroup congruence on $S$. Let $(x, y) \in \mu$ then $(x)_{\mu}^{\delta}=(x)_{\mu}^{-1}=(y)_{\mu}^{-1}=\left(y^{\delta}\right)_{\mu}$ and clearly $\left(x^{\delta}\right)_{\mu^{\delta}}=\left(y^{\delta}\right)_{\mu^{\delta}}$. Thus by $(\mu 1)$ we have that $x^{\delta}=y^{\delta}$. We consider that $(x)_{\xi} \in S / \xi$ since $x^{\delta}=y^{\delta}$ so that $\left(x^{\delta}\right)_{\xi}$ and $\left(y^{\delta}\right)_{\xi}$ are both inverse in $S / \mu$ of $(x)_{\xi}$ and so $\left(x^{\delta}\right)_{\xi}=(x)_{\xi}^{-1}=(y)_{\xi}^{-1}=\left(y^{\delta}\right)_{\xi}$ by the uniqueness of inverse in $S / \xi$. Thus we obtain $(x, y) \in \xi$, that is, $\mu \subseteq \xi$ which shows that $\mu$ is the smallest inverse semigroup congruence on $S$.

$(2) \Longrightarrow(1)$ Suppose that the congruence $\mu$ has the properties $(\mu 1)$ and $(\mu 2)$ we shall prove that $\left|V_{S^{\delta}}(x)\right|=1$ for any $x \in S$. Let $y^{\delta} \in V_{S^{\delta}}(x)$ then by $(\mu 2)$ we know that $\left(x^{\delta}\right)_{\mu}$ and $\left(y^{\delta}\right)_{\mu}$ are both inverse in $S / \mu$ of $(x)_{\mu}$. By the uniqueness of inverse in $S / \mu$ we obtain that $\left(x^{\delta}\right)_{\mu}=\left(y^{\delta}\right)_{\mu}$ and $\left(x^{\delta}\right)_{\mu_{\delta}}=\left(x^{\delta}\right)_{\mu_{\delta}}$. Since $\mu_{\delta}$ is the identity on $S^{\delta}$ by $(\mu 1)$, so $x^{\delta}=y^{\delta}$, that is, $\left|V_{S^{\delta}}(x)\right|=1$ for any $x \in S$ which shows $S^{\delta}$ is an inverse ${ }^{\delta}$ transversal of $S$. Since $S^{\delta}$ is a po-semigroup, so $S^{\delta}$ is also an inverse ${ }^{\delta}$ po-transversal of $S$ and it is fundamental by $(\mu 1)$.

Corollary 1.5. Let $S^{\delta}$ be a po-subsemigroup of the ORB-(resp.POR-) semigroup $S$ with the mapping $\delta$ is surjective from $S$ to $S^{\delta}$ such that $x^{\delta} \in V_{S^{\delta}}(x)$ for $x \in S$, then the following statements are equivalent:

(1) $S^{\delta}$ is a fundamental inverse ${ }^{\delta}$ po-transversal of $S$;

(2) The equivalence relation

$$
\mu=\left\{(x, y) \in \mathcal{H} \mid x^{\delta} e x^{\delta \delta}=y^{\delta} e y^{\delta \delta} \text { for any } e \in E\left(S^{\delta}\right)\right\}
$$

has the following properties:

$(\mu 1) \mu_{\delta}=\mu \cap\left(S^{\delta} \times S^{\delta}\right)$ is the maximum idempotent-separating congruence on $S^{\delta}$ and $\mu_{\delta}$ is the identity congruence on $S^{\delta}$;

$(\mu 2) \mu$ is the smallest inverse semigroup congruence on $S$. 


\section{The existence of inverse ${ }^{\delta}$ po-transversals for some classes of po-semigroups}

In the proof of Theorem 1.2, we may see that the anti-homomorphism $\delta$ is very important. We think thus that if there exists an anti-homomorphism $\delta$ from some regular po-semigroup $S$ to some po-subsemigroup $S^{\delta}$ of $S$ then it is possible that $S$ has an inverse ${ }^{\delta}$ po-transversal. In fact, we may show that it is true for some po-semigroup.

Theorem 2.1. Let $S$ be an order orthodox semigroup with the biggest inverses (for short, OOB-semigroup). Then $S$ has the weakly multiplicative inverse ${ }^{\delta}$ potransversal as following:

$$
S^{\delta}=\left\{x^{\delta} \in V(x) \mid x^{\delta} \hat{=} x^{o} \in(x)_{\nu}^{-1} \text { for any } x \in S\right\}
$$

with the mapping $\delta$ is an anti-homomorphism from $S$ to $S^{\delta}$ and the mapping $\delta \delta$ denoted by $x^{\delta \delta}=\left(x^{\delta}\right)^{\delta} \in(x)_{\nu}$ for any $x \in S$ is a homomorphism from $S$ to $S^{\delta}$. Here,

$$
\nu=\left\{(x, y) \in S \times S \mid V(x)=V(y) \text { and } x^{o}=y^{o}\right\}
$$

is the smallest inverse semigroup congruence on $S$ where $a^{o} \in V(a)$ for any $a \in$ $S$ and $a^{o}$ is the biggest inverse of $a$.

Proof. Let $S$ be an OOB-semigroup. We know that equivalence relation

$$
\nu=\{(x, y) \in S \times S \mid V(x)=V(y)\}
$$

is the smallest inverse semigroup congruence on $S$ by [6, VI.1 Theorem 1.12]. We denote the biggest inverse of $x$ by $x^{o}$ for $x \in S$. Let the equivalence relation

$$
\tau=\left\{(x, y) \in S \times S \mid x^{o}=y^{o}\right\},
$$

then we have the following results. $(\alpha) \nu=\tau \cap \nu$.

Let $(x, y) \in \tau$ and $z \in S$ then by $S$ being orthodox we have $(z x)^{o}=x^{o} z^{o}=$ $y^{o} z^{o}=(z y)^{o}$ so $(z x, z y) \in \tau$. Similarly $(x z, y z) \in \tau$, that is, $\tau$ is a congruence on $S$. Let $\xi=\tau \cap \nu$ then $\xi$ is also a congruence on $S$. We denote the $\xi$-class (resp. $\nu$-class) of containing $x$ by $(x)_{\xi}$ (resp. $\left.(x)_{\nu}\right)$. Similar to Theorem 1.2, we may show that $\xi$ is also an inverse semigroup congruence on $S$ and $\xi \subseteq \nu$. By the smallest property of $\nu$ we have $\xi=\tau \cap \nu=\nu$.

( $\beta)(\forall x \in S)(x)_{\nu}^{-1}=\left(x^{o}\right)_{\nu},(x)_{\nu}=\left(x^{o o}\right)_{\nu}$.

Since $(x)_{\nu}^{-1}$ is the unique of $(x)_{\nu}$ and clearly $(x)_{\nu}^{-1}=\left(x^{o}\right)_{\nu}$ by $x^{o} \in V(x)$. If $(x)_{\nu}^{-1}=\left(x^{o}\right)_{\nu}=\left(y^{o}\right)_{\nu}$ then $x^{o}=y^{o}$ by $(\alpha)$. By $(x)_{\nu}^{-1}=\left(x^{o}\right)_{\nu}$ imply $(x)_{\nu}=\left((x)_{\nu}^{-1}\right)^{-1}=\left(\left(x^{o}\right)_{\nu}\right)^{-1}=\left(x^{o o}\right)_{\nu}$ is also unique.

$(\gamma)(\forall x \in S)$ If $y \in(x)_{\nu}$ then $y^{o}=x^{o}$ and $y^{o o}=x^{o o}$.

By $(\beta)$ for $x \in S, x^{o o} \in(x)_{\nu}$, if $y \in(x)_{\nu}$ then $(x)_{\nu}=(y)_{\nu}$, by $(\alpha) \nu=\tau \cap \nu$ so $x^{o}=y^{o}$ and imply $x^{O O}=y^{o O}$.

( $\delta) S^{\delta}=\left\{x^{\delta} \mid x^{\delta} \hat{=} x^{o} \in(x)_{\nu}^{-1}\right.$, for any $\left.x \in S\right\}$ is a po-semigroup with $\delta$ is an anti-homomorphism from $S$ to $S^{\delta}$ and $\delta \delta$ is a homomorphism.

By $(\beta)$ and $(\gamma)$ for any $x \in S$ there is the inverse $x^{o} \in(x)_{\nu}^{-1}$. For the inverse $x^{o} \in(x)_{\nu}^{-1}$ we denote by $x^{\delta} \hat{=} x^{o} \in(x)_{\nu}^{-1}$ then $\delta$ is a mapping from $S$ onto $S^{\delta}$ 
and $x^{\delta} y^{\delta}=(y x)^{\delta} \in(y x)_{\nu}^{-1}, x^{\delta \delta} y^{\delta \delta}=(x y)^{\delta \delta} \in(x y)_{\nu}$ by $(\beta)$. So we have that $S^{\delta}$ is a po-semigroup and statement $(\delta)$ holds.

( $\epsilon) S^{\delta}$ is an inverse ${ }^{\delta}$ po-transversal of $S$.

Let the mapping $\phi: S^{\delta} \longrightarrow S / \nu$ denoted by $x^{\delta} \longmapsto(x)_{\nu}$, that is, $x^{\delta} \phi=(x)_{\nu}$. If $(x)_{\nu}=(y)_{\nu}$ then $x^{\delta}=y^{\delta}$ and for any $(x)_{\nu} \in S / \nu$ there is $x^{\delta} \in(x)_{\nu}$ such that $x^{\delta} \phi=(x)_{\nu}$. Let $x^{\delta}, y^{\delta} \in S^{\delta}$ then $\left(x^{\delta} y^{\delta}\right) \phi=(y x)^{\delta} \phi=(y x)_{\nu}=(y)_{\nu} \cdot(x)_{\nu}=$ $y^{\delta} \phi \cdot x^{\delta} \phi$. Thus we know that $\phi$ is an anti-isomorphism from $S^{\delta}$ to $S / \nu$. So $S^{\delta}$ is also an inverse po-semigroup by $S / \nu$ being inverse. Clearly, $x^{\delta}$ is the unique element in $V_{S^{\delta}}(x)$, so $S^{\delta}$ is an inverse ${ }^{\delta}$ po-transversal of $S$. By lemma 1.1(7), $S^{\delta}$ is weakly multiplicative.

Since the principally ordered orthodox semigroups (for short, POO-semigroups) are OOB-semigroups, we have

Corollary 2.2. Let $S$ be an POO-semigroup. Then $S$ has the weakly multiplicative inverse ${ }^{\delta}$ po-transversal

$$
S^{\delta}=\left\{x^{\delta} \in V(x) \mid x^{\delta} \hat{=} x^{o} \in(x)_{\nu}^{-1} \text { for any } x \in S\right\}
$$

with the mapping $\delta$ is an anti-homomorphism from $S$ to $S^{\delta}$ and the mapping $\delta \delta$ denoted by $x^{\delta \delta}=\left(x^{\delta}\right)^{\delta} \in(x)_{\nu}$ for $x \in S$ is a homomorphism from $S$ to $S^{\delta}$. Here,

$$
\nu=\left\{(x, y) \in S \times S \mid V(x)=V(y) \text { and } x^{o}=y^{o}\right\}
$$

is the smallest inverse semigroup congruence on $S$ where $x^{o}$ as above for $x \in S$.

It is well known that for $O O B$-semigroup $S . S^{o}$ isn't necessarily an inverse ${ }^{\circ}$ transversal of $S$. Theorem 2.1 and Corollary 2.2 solved the existence problems of inverse ${ }^{\delta}$ po-transversals of $O O B$-semigroups and $P O O$-semigroups.

Here we list the following basic facts on ORB-semigroups, which will be used in following theorems, the reader can consult [1]-[3] for more details.

Lemma 2.3. Let $S$ be a ORB-semigroup and $S^{o}=\left\{x^{o} \mid \forall x \in S\right\}$ then we have

(1) $(\forall e \in E(S)) e \leq e^{o} \leq\left(e^{o}\right)^{2}, e=e e^{o} e^{o o} e=e e^{o o} e^{o} e, e^{o o} \in V(e) \cap E(S)$ and $e^{o} \in E(S) \Leftrightarrow e^{o}=e^{o o}$;

(2) $(\forall x \in S) x \leq x^{o o}, x^{o}=x^{o o o}$ and $\left(x x^{o}\right)^{o}=x^{o o} x^{o},\left(x^{o} x\right)^{o}=x^{o} x^{o o}$;

(3) $(\forall x, y \in S) x \mathcal{R} y$ if and only if $x x^{o}=y y^{o} ; x \mathcal{L} y$ if and only if $x^{o} x=y^{o} y$ and $x^{o} x$ (resp. $\left.x x^{o}\right)$ is the biggest idempotent in $(x)_{\mathcal{L}}\left(\right.$ resp. $\left.(x)_{\mathcal{R}}\right)$

(4) if $\mathcal{R}$ and $\mathcal{L}$ are weakly regular $($ see $[1])$ then

$(\alpha)(\forall e \in E(S)) e^{o}=e^{o o} \in E(S)$;

$(\beta)(\forall x \in S) V(x) \cap S^{o}=\left\{x^{o}\right\}$;

$(\nu)$ if $S^{o}$ is a po-subsemigroup of $S$ then $S^{0}$ is an inverse ${ }^{o}$ po-transversal of $S$

Lemma 2.4. Let $S$ be ORB-semigroup on which $\mathcal{R}$ and $\mathcal{L}$ are strongly regular then

$$
(\forall x, y \in S)(x y)^{o}=\left(x^{o} x y\right)^{o} x^{o}=y^{o}\left(x y y^{o}\right)^{o} .
$$


Proof. Let $x, y \in S$ then $x y \mathcal{L} x^{o} x y$ so that $(x y)^{o} x y=\left(x^{o} x y\right)^{o} \cdot x^{o} x y$ by Lemma 2.3 (3). Since $(x y)(x y)^{o} \mathcal{R} x y\left(x^{o} x y\right)^{o} x^{o}$. Similarly,

$$
x y(x y)^{o}=x y(x y)^{o}\left(x y(x y)^{o}\right)^{o}=x y\left(x^{o} x y\right)^{o} x^{o}\left(x y \cdot\left(x^{o} x y\right)^{o} x^{o}\right)^{o} .
$$

Since $x x^{o}, x y\left(x^{o} x y\right)^{o} x^{o} \in E(S)$ and

$$
x y\left(x^{o} x y\right)^{o} x^{o}=x x^{o} x y\left(x^{o} x y\right)^{o} x^{o}=x y\left(x^{o} x y\right)^{o} x^{o} x x^{o}
$$

so that $x y\left(x^{o} x y\right)^{o} \cdot x^{o} \leq_{n} x x^{o}$. By $\mathcal{R}$ and $\mathcal{L}$ being strongly regular we have $x y(x y)^{o}=x y\left(x^{o} x y\right)^{o} x^{o}\left(x y\left(x^{o} x y\right)^{o} x^{o}\right)^{o} \leq x x^{o}\left(x x^{o}\right)^{o}=x x^{o}$. Thus $x^{o} x y(x y)^{o}$ $\leq x^{o}$. Since $\left(x^{o} x y\right)^{o} x^{o} \in V(x y)$, so $\left(x^{o} x y\right)^{o} x^{o} \leq(x y)^{o}$. Then we have

$$
x y(x y)^{o}=x y\left(x^{o} x y\right)^{o} x^{o} x y(x y)^{o} \leq x y\left(x^{o} x y\right)^{0} x^{0} \leq x y(x y)^{o} .
$$

Consequently,

$$
x y(x y)^{o}=x y\left(x^{o} x y\right)^{o} x^{o}
$$

so that

$$
\begin{aligned}
(x y)^{o} & =(x y)^{o} x y(x y)^{o}=(x y)^{o} x y\left(x^{o} x y\right)^{o} x^{o}=\left(x^{o} x y\right)^{o} x^{o} x y \cdot\left(x^{o} x y\right)^{o} x^{o} \\
& =\left(x^{o} x y\right)^{o} x^{o} .
\end{aligned}
$$

Similarly, we have $(x y)^{o}=y^{o}\left(x y y^{o}\right)^{o}$.

Now we may prove the following theorem. This theorem will solve the existence of multiplicative inverse ${ }^{\delta}$ po-transversals for the ORB- and PORsemigroups on which $\mathcal{R}$ and $\mathcal{L}$ are strongly regular.

Theorem 2.5. Let $S$ be an ORB-semigroup on which $\mathcal{R}$ and $\mathcal{L}$ are strongly regular then $S^{\circ}$ is necessarily a multiplicative inverse ${ }^{\circ}$ po-transversal of $S$.

Proof. We show first that $S^{o}$ is a quasi-ideal of $S$ and so that $S^{o}$ is a posubsemigroup of $S$ since $S$ is regular. Let $a, b \in S^{0}, x \in S$ and $y=a x b$ then $a=a^{o o}$ and $b=b^{o o}$ by Lemma 2.3 (2). By Lemma 2.4 we have $y y^{o}=$ $y y^{o} a a^{o}=a a^{o} y y^{o}$ so that $y y^{o} \leq_{n} a a^{o}$. Similarly, $y^{o} y \leq_{n} b^{o} b$. By $\mathcal{R}$ and $\mathcal{L}$ being strongly regular and Lemma $2.3(2)$ we obtain $\left(y y^{o}\right)^{o} y y^{o}=y^{o o} y^{o} y y^{o}=$ $y^{o o} y^{o} \leq\left(a a^{o}\right)^{o} a a^{o}=a a^{o}$. Therefore we have $y^{o o} y^{o} y \leq a a^{o} y=a a^{o} a x b=a x b=$ $y=y y^{o} y \leq y^{o o} y^{o} y$ by Lemma 2.3 (2). Consequently, $y=y^{o o} y^{o} y$ and similarly $y=y y^{o} y^{o o}$. Thus we obtain $y=y y^{o} y=y^{o o} y^{o} y y^{o} y=y^{o o} y^{o} y=y^{o o} y^{o} y y^{o} y^{o o}=$ $y^{o o} y^{o} y^{o O}=y^{o o} \in S^{o}$, which shows that $S^{o}$ is a quasi-ideal of $S$.

Now we know immediately that $S^{o}$ is an inverse ${ }^{o}$ po-transversal of $S$ with the mapping $o$ is surjective from $S$ to $S^{o}$ by lemma 2.3(4) $(\nu)$. We may prove that $S^{o}$ is multiplicative. In fact, by lemma $2.3(4)(\alpha) e^{o} \in E(S)$ for any $e \in E(S)$, then by lemma 1.1(6) and upper result we have that $S^{o}$ is multiplicative.

Corollary 2.6. Let $S$ be a POR-semigroup on which $\mathcal{R}$ and $\mathcal{L}$ are strongly regular then $S^{o}=\left\{x^{o} \in V(x) \mid x^{o}=x^{*} x x^{*}\right.$ for any $\left.x \in S\right\}$ is necessarily a multiplicative inverse ${ }^{\circ}$ po-transversal of $S$. 


\section{The structures of ORB- and POR-semigroups}

By the results in Section 2, on the understanding that $\mathcal{R}$ and $\mathcal{L}$ are strongly regular, the following structure theorem is may obtained by Blyth and McFadden in [4].

Theorem 3.1. Let $S$ be a ORB-semigroup on which $\mathcal{R}$ and $\mathcal{L}$ are strongly regular. Let $S^{o}=\left\{x^{o} \mid \forall x \in S\right\}, I=\left\{x x^{o} \mid \forall x \in S\right\}$ and $\wedge=\left\{x^{o} x \mid \forall x \in S\right\}$, then $S$ is ordering ${ }^{o}$-isomorphic to $W$ (i.e. $x^{o} \phi=(x \phi)^{\circ}$ for $x \in S$ and the ordering isomorphism $\phi$ ) as following.

$$
W=\left\{(e, a, f) \in I \times S^{0} \times \Lambda \mid e^{0}=a a^{-1}, f^{0}=a^{-1} a\right\}
$$

with the Cartesian order

$$
(e, a, f) \preceq(g, b, h) \Longleftrightarrow e \leq g, a \leq b, f \leq h
$$

where multiplicative in $W$ is defined by

$$
(e, a, f)(g, b, h)=\left(e a f g a^{-1}, a f g b, b^{-1} f g b h\right) .
$$

Proof. We need only to prove that $S$ is ordering ${ }^{0}$-isomorphic to $W$. The rests are obtained by Blyth and McFadden in [4]. Let the mapping

$$
\phi: S \longrightarrow W, x \phi=\left(x x^{0}, x^{00}, x^{0} x\right)=\left(e_{x}, x^{00}, f_{x}\right)
$$

where $e_{x}=x x^{0}$ and $f_{x}=x^{0} x$ for any $x \in S$, then $\phi$ is an algebraic isomorphism by [4]. It's inverse $\phi^{-1}$ is given by $(e, a, f) \phi^{-1}=e a f$ for each $(e, a, f) \in W$. To show that $\phi$ is isotone, suppose that $x \leq y$ in $S$ then

$$
\begin{array}{rlrl}
x \leq y & \Longrightarrow x^{0} x \leq y^{0} y & & \text { (by } \mathcal{L} \text { is regular) } \\
& \Longrightarrow\left(x^{0} x\right)\left(x^{0} x\right)^{0} \leq\left(y^{0} y\right)\left(y^{0} y\right)^{0} & \text { (by } \mathcal{R} \text { is regular) } \\
& \Longrightarrow x^{0} x^{00} \leq y^{0} y^{00} & & \text { (by lemma 1.1(1) and }(2)) .
\end{array}
$$

Similarly, $x x^{0} \leq y y^{0}$ and $x^{00} x^{0} \leq y^{00} y^{0}$, therefore $x^{00}=x^{00} x^{0} x x^{0} x^{00} \leq$ $y^{00} y^{0} y y^{0} y^{00}=y^{00}$. We conclude that $\left(e_{x}, x^{00}, f_{x}\right)=x \phi \preceq y \phi=\left(e_{y}, y^{00}, f_{y}\right)$, that is, $\phi$ is isotone and is therefore an isomorphism of po-semigroups.

By [4] we know $(x \phi)^{0}=\left(e_{x}, x^{00}, f_{x}\right)^{0}=\left(e_{x^{0}}, x^{0}, f_{x^{0}}\right)$. By Lemma 1.1 (1) and (2) we compute that $x^{0} \phi=\left(e_{x^{0}}, x^{0}, f_{x^{0}}\right)$ so imply $x^{0} \phi=(x \phi)^{0}$.

The following result is a generalization of Theorem 2.6 in [7].

Corollary 3.2. Let $S$ be a ORB-semigroup on which the order $\leq$ is natural. If $\mathcal{R}$ and $\mathcal{L}$ are regular then $S^{\circ}=\left\{x^{o} \in V(x) \mid \forall x \in S\right\}$ is a multiplicative inverse ${ }^{o}$ po-transversal of $S$ and $S$ is ordering ${ }^{{ }^{o}}$-isomorphic to $W$ as above.

Proof. We need only to notice that when the order $\leq$ is natural then $\mathcal{R}$ and $\mathcal{L}$ are regular if and only if $\mathcal{R}$ and $\mathcal{L}$ are strongly regular. Thus we immediately obtain that the result holds by Theorem 3.1 .

For the POR-semigroups we have the following results. 
Theorem 3.3. Let $S$ be a POR-semigroup on which $\mathcal{R}$ and $\mathcal{L}$ are strongly regular then

(1) $S$ has the multiplicative inverse ${ }^{0}$ po-transversal $S^{0}=\left\{x^{0} \in V(x) \mid \forall\right.$ $x \in S\}$ with the mapping o is surjective from $S$ to $S^{0}$;

(2) As an ORB-semigroup then $S$ is ordering ${ }^{\circ}$-isomorphic to $W$ as above;

(3) Let $\phi$ is an ordering ${ }^{\circ}$-isomorphism from $S$ to $W$, then $W$ can be principally ordered such that $W$ becomes a POR-semigroup and $\phi$ becomes an ordering *-isomorphism from $S$ to POR-semigroup $W$.

Proof. By Theorem 3.1 the statement (1) and (2) hold. We need only to show the statement (3). Since $\phi$ is an ordering isomorphism, then $\phi^{-1}$ is also and

$$
x y x \leq x \Longleftrightarrow(x y x) \phi \preceq x \phi(\forall x, y \in S) .
$$

Since for each $x \in S$ there exists $x^{*}=\max \{y \in S \mid x y x \leq x\}$. So for each $\left(e_{x}, x^{o o}, f_{x}\right) \in W$ there also exists

$$
\begin{aligned}
\left(e_{x}, x^{o o}, f_{x}\right)^{*} & =\max \left\{\left(e_{y}, y^{o o}, f_{y}\right) \in W \mid\left(e_{x}, x^{o o}, f_{x}\right)\left(e_{y}, y^{o o}, f_{y}\right)\left(e_{x}, x^{00}, f_{x}\right)\right. \\
& \left.=\left(e_{x y x},(x y x)^{00}, f_{x y x}\right) \preceq\left(e_{x}, x^{00}, f_{x}\right)\right\} .
\end{aligned}
$$

In fact, by Lemma in [2] we compute that $\left(e_{x}, x^{00}, f_{x}\right)^{*}=\left(e_{x^{*}},\left(x^{*}\right)^{00}, f_{x^{*}}\right)$ and $\left(e_{x}, x^{00}, f_{x}\right)^{0}=\left(e_{x^{0}},\left(x^{0}\right)^{00}, f_{x^{\circ}}\right)$. Therefore we know that $W$ can be principally ordered by the ordering isomorphism $\phi$. In such a way, if $x \in$ $S, x \phi=\left(e_{x}, x^{00}, f_{x}\right)$ then clearly $(x \phi)^{*}=\left(e_{x}, x^{00}, f_{x}\right)^{*}=\left(e_{x^{*}},\left(x^{*}\right)^{00}, f_{x^{*}}\right)$ and $x^{*} \phi=\left(e_{x^{*}},\left(x^{*}\right)^{00}, f_{x^{*}}\right)$, that is, $x^{*} \phi=(x \phi)^{*}$.

In closing this note, we point that the POR-semigroup $M$ in Example 1 has multiplicative inverse ${ }^{0}$ po-transversal

$$
M^{0}=\left\{\left[x, y, p_{k}+k-1\right] \in M \mid(x, y) \in L^{[2]}, p \in Z\right\} .
$$

In fact, $E\left(M^{0}\right)=\left\{[x, y, k-1] \mid(x, y) \in L^{[2]}\right\}$ is a semilattice and $M^{0}$ is an quasi-ideal of $M$. The proof here is omitted.

\section{References}

[1] T. S. Blyth and G. A. Pinto, On idempotent-generated subsemigroups of principally ordered regular semigroups, Semigroup Forum 65 (2003), 1-12.

[2] _ Idempotents in principally ordered regular semigroups, Communications in Algebra 19 (1991), 1549-1563.

[3] _ On ordered regular semigroup with biggest inverses, Semigroup Forum 54 (1997), $154-165$.

[4] T. S. Blyth and R. McFadden, Regular semigroups with a multiplicative inverse transversal, Proc. Rog. Soc. Edinburgh 92A (1982), 253-270.

[5] Z. Gao, Naturally ordered abundant semigroups with adequate transversals, PU. M. A 14 (2003), no. 1-2, 35-50.

[6] J. M. Howie, An Introduction to Semigroups Theory, Academic Press. London, 1976.

[7] S. Tatsuhiko, Naturally ordered regular semigroups with maximum inverses, Roc. Edinburgh Math. Sec. 32 (1989), 33-39. 
Zhenlin GaO

Science College of University of Shanghai for Science and Technology

Shanghai 200093, ChinA

E-mail address: zlgao@sina.com

GuiJie ZhANG

Science College of University of Shanghai for Science and Technology

Shanghai 200093, ChinA

E-mail address: dreamerzgj@163.com 